\title{
Improving entanglement concentration of Gaussian states by local displacements
}

\author{
Jaromír Fiurášek \\ Department of Optics, Palacký University, 17. listopadu 12, 77146 Olomouc, Czech Republic
}

\begin{abstract}
We investigate entanglement concentration of continuous-variable Gaussian states by local singlephoton subtractions combined with local Gaussian operations. We first analyze the local squeezingenhanced entanglement concentration protocol proposed very recently by Zhang and van Loock [eprint: arXiv:1103.4500 (2011)] and discuss the mechanism by which local squeezing before photon subtraction helps to increase the entanglement of the output state of the protocol. We next show that a similar entanglement improvement can be achieved by using local coherent displacements instead of single-mode squeezing.
\end{abstract}

PACS numbers: 03.67.Mn,03.67.Hk, 42.50.Dv

\section{INTRODUCTION}

Distillation of continuous-variable entanglement is fundamentally limited by a no-go theorem which states that experimentally accessible entangled Gaussian states cannot be distilled by local Gaussian operations and classical communication [1-3]. In order to increase entanglement of a Gaussian state such as two-mode squeezed vacuum by local operations, one thus has to resort to non-Gaussian operations [4]. A prominent example of such an operation is the photon subtraction, that has been proposed for continuous-variable entanglement concentration in a seminal paper by Opatrný et al. [5]. Since then, the photon subtraction has been recognized as a crucial tool for implementing many tasks in optical continuous-variable quantum information processing. Besides entanglement concentration [6-10], this includes generation of Schrödinger cat-like states by subtracting photons from single-mode squeezed vacuum state [11 14], generation of arbitrary single-mode states of light beams [15, 16], implementation of quantum gates for qubits encoded into superpositions of two coherent states [17], and probabilistic noiseless amplification of coherent states of light [18-24].

Although Gaussian operations alone are useless for distillation of Gaussian entanglement, it has been recently shown by Zhang and van Loock 25] that the performance of entanglement concentration via local singlephoton subtraction can be enhanced by suitable local squeezing operations. In this paper we further investigate this intriguing concept and clarify the origin of enhancement of entanglement concentration due to the local pre-squeezing. We next show that the same effect can be achieved also by local displacements, which are much easier and experimentally more feasible operations than squeezing. Indeed, the combination of photon subtraction and coherent displacement has been recently successfully experimentally utilized to generate arbitrary superpositions of squeezed vacuum and squeezed singlephoton states from squeezed vacuum [16]. Coherent displacement can be implemented by mixing the signal beam with a weak coherent laser beam on a highly unbalanced beam splitter. In contrast, squeezing is much more exper- imentally challenging and resource demanding operation. Squeezing in cavity-based optical parametric amplifier would require efficient injection of the pulsed signal beam in the cavity as well as efficient retrieval of the squeezed beam which is a nontrivial task. Squeezing based on a single passage through a nonlinear crystal pumped by a strong pulsed laser still requires careful modematching and may suffer from noise due to spontaneous parametric down-conversion producing pairs of photons in many modes. Alternatively, one can employ an off-line generated auxiliary squeezed vacuum state combined with interference on an unbalanced beam splitter, homodyne detection and feedforward [26, 27]. This squeezing technique was successfully experimentally tested, however, the operation is imperfect and inevitably adds some extra noise due to finite squeezing of the auxiliary state.

The rest of the paper is structured as follows. In Section II we present a simple analysis of the local squeezingenhanced entanglement concentration which reveals the origin of the entanglement enhancement in this scheme. In Section III we describe the displacement-enhnaced entanglement concentration procedure and we analytically determine the optimal displacement and success probability scaling in the limit of pure weakly squeezed input states, where the scheme achieves the maximum entanglement enhancement. We also numerically calculate the optimal local displacements maximizing entanglement of the distilled state for both pure and mixed Gaussian twomode squeezed states. Finally, Section IV contains brief summary and conclusions.

\section{SQUEEZING-ENHANCED ENTANGLEMENT CONCENTRATION}

The considered entanglement concentration scheme is depicted in Fig. 1(a). The entangled two-mode squeezed state is distributed from a source via some quantum channel to the two recipients traditionally called Alice and Bob who attempt to conditionally increase the entanglement of the shared state by local photon subtraction, which is a specific example of a quantum filter. The whole scheme is thus a typical example of a Procrustean entanglement concentration protocol [28]. In practice, 

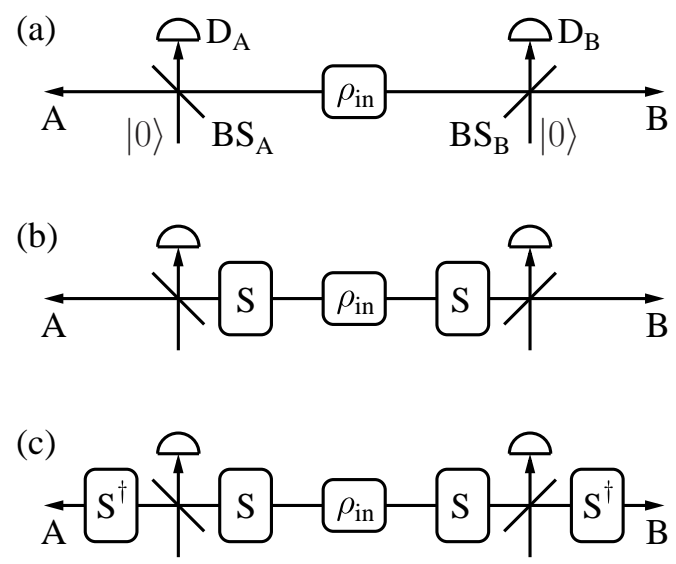

FIG. 1: (a) Entanglement concentration by single-photon subtraction. $\mathrm{BS}_{A}$ and $\mathrm{BS}_{B}$ are highly unbalanced beam splitters, $\hat{\rho}_{\text {in }}$ is the shared (generally mixed) entangled state, and $\mathrm{D}_{A}$ and $\mathrm{D}_{B}$ denote single-photon detectors. (b) Entanglement concentration improved by local single-mode suqeezing $\hat{S}$. The state can be un-squeezed after concentration by inverse local operations $\hat{S}^{\dagger}$ without changing its entanglement (c).

the photon subtraction is accomplished by means of a highly unbalanced beam splitter that reflects a tiny part of the incoming beam on a single-photon detector APD. A successful photon subtraction is heralded by a click of the detector. Alice and Bob exchange their measurement results via classical communication line and the entanglement concentration succeeds if they both register detector clicks. In the limit of very low beam splitter reflectance, the resulting operation can be described by action of annihilation operators $\hat{a}$ and $\hat{b}$ on modes $\mathrm{A}$ and $\mathrm{B}$, respectively, even if the detectors have non-unit detection efficiency and cannot distinguish number of photons.

It is instructive to first investigate concentration of a pure two-mode squeezed vacuum state

$$
|\psi\rangle=\sqrt{1-\lambda^{2}} \sum_{n=0}^{\infty} \lambda^{n}|n n\rangle,
$$

where $|n\rangle$ denotes Fock state, $|n n\rangle$ is a short-hand notation for $|n\rangle|n\rangle$, and $\lambda=\tanh r$, where $r$ stands for the two-mode squeezing constant. The non-normalized state after local photon subtractions can be expressed as

$$
\hat{a} \otimes \hat{b}|\psi\rangle=\sqrt{1-\lambda^{2}} \sum_{n=0}^{\infty}(n+1) \lambda^{n+1}|n n\rangle .
$$

Alice and Bob may amend the entanglement concentration protocol by local squeezing of modes A nad B prior to the photon subtraction [25], c.f. Fig. 1(b). The filtering operation then reads $\hat{a} \otimes \hat{b} \hat{S}_{A}(s) \otimes \hat{S}_{B}(s)$, where $\hat{S}_{M}$ is a single-mode squeezing operation on mode M with squeezing constant $s$. In order to clarify why the pre-squeezing can be useful, we apply an inverse unitary squeezing operation $\hat{S}_{A}^{\dagger}(s) \otimes \hat{S}_{B}^{\dagger}(s)$ to the concentrated state which does not change its entanglement, see Fig. 1(c). The overall filtering operation $\hat{F}_{A B}$ then reads

$$
\hat{F}_{A B}=\hat{S}_{A}^{\dagger}(s) \hat{a} \hat{S}_{A}(s) \otimes \hat{S}_{B}^{\dagger}(s) \hat{b} \hat{S}_{B}(s) .
$$

The squeezing transforms the annihilation operators into linear combination of annihilation and creation operators, and we have

$$
\hat{F}_{A B}=\left[\hat{a} \cosh (s)+\hat{a}^{\dagger} \sinh (s)\right] \otimes\left[\hat{b} \cosh (s)+\hat{b}^{\dagger} \sinh (s)\right] .
$$

We can thus see that the local pre-squeezing effectively converts the photon subtraction into a coherent combination of photon subtraction and photon addition.

Let us now investigate what happens in the limit of weak two-mode squeezing, $\lambda \ll 1$. We can write

$$
\hat{F}_{A B}|\psi\rangle=\hat{F}_{A B}(|00\rangle+\lambda|11\rangle)+O\left(\lambda^{2}\right),
$$

where $O\left(\lambda^{2}\right)$ indicates small terms of the order of $\lambda^{2}$. After some algebra we get

$$
\begin{aligned}
\hat{F}_{A B}|\psi\rangle= & \lambda \cosh ^{2}(s)|00\rangle+\sinh ^{2}(s)|11\rangle+2 \lambda \sinh ^{2}(s)|22\rangle \\
& +\frac{\lambda}{\sqrt{2}} \sinh (2 s)(|02\rangle+|20\rangle)+O\left(\lambda^{2}\right),
\end{aligned}
$$

If we set $\tanh (s)=\sqrt{\lambda}$, then we obtain

$$
\hat{F}|\psi\rangle \approx \lambda(|00\rangle+|11\rangle)+O\left(\lambda^{3 / 2}\right) .
$$

Note that the amplitude of the states $|02\rangle$ and $|20\rangle$ is suppressed by a factor of $\sqrt{\lambda}$ with respect to the amplitude of the dominant term $|00\rangle+|11\rangle$. In the limit of very small $\lambda$ the result of entanglement concentration is a maximally entangled state of two qubits hence the protocol can extract one e-bit of entanglement from arbitrarily weakly squeezed initial state. The success probability of the protocol scales as $P_{\text {succ }} \propto \lambda^{2}$ for $\lambda \ll 1$. The squeezing enhanced protocol should be contrasted with the ordinary concentration by local photon subtraction that in the limit $\lambda \ll 1$ provides only very weakly entangled state $|00\rangle+2 \lambda|11\rangle$.

\section{DISPLACEMENT-ENHANCED ENTANGLEMENT CONCENTRATION}

We next show that similar improvement in the amount of extracted entanglement can be obtained if we replace the local squeezing operations with local displacements $\hat{D}_{A}(\alpha)$ and $\hat{D}_{B}(\beta)$, see Fig. 2(a). Similarly as above, we assume that the displacement is undone after photon subtraction by inverse displacements $\hat{D}_{A}^{\dagger}(\alpha)$ and $\hat{D}_{B}^{\dagger}(\beta)$, c.f. Fig. 2(b). The resulting local quantum filter reads

$$
\hat{G}_{A B}=\hat{D}_{A}^{\dagger}(\alpha) \hat{a} \hat{D}_{A}(\alpha) \otimes \hat{D}_{B}^{\dagger}(\beta) \hat{b} \hat{D}_{B}(\beta) .
$$



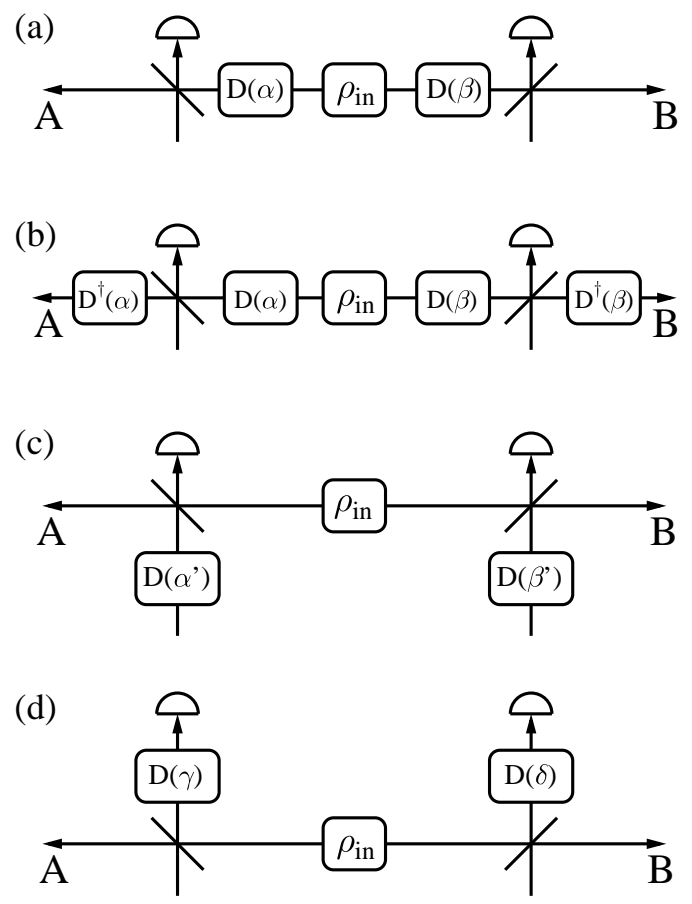

FIG. 2: Entanglement concentration scheme combining local displacements and photon subtraction (a). Applying local unitary inverse displacement operations on the state after concentration does not change its entanglement (b). Instead of displacing the signal modes one can equivalently inject weak coherent states into the auxiliary ports of the unbalanced beam splitters (c) or displace the beams impinging on single-photon detectors (d).

The local displacements transform the photon subtraction into a coherent superposition of photon subtraction and identity operation and we have

$$
\hat{G}_{A B}=(\hat{a}+\alpha) \otimes(\hat{b}+\beta) .
$$

Let us again consider the limit $\lambda \ll 1$. We obtain

$\hat{G}_{A B}|\psi\rangle=(\alpha \beta+\lambda)|00\rangle+\lambda(\alpha|10\rangle+\beta|01\rangle)+\lambda \alpha \beta|11\rangle+O\left(\lambda^{2}\right)$.

The apparently dominant vacuum term corresponds to a separable state, but it can be completely eliminated by a destructive interference provided that $\alpha \beta=-\lambda$. The maximal entanglement is then obtained if the displacements are real and symmetric,

$$
\alpha=-\beta=\sqrt{\lambda}
$$

If we insert $\alpha$ and $\beta$ given by Eq. (10) into (9) we get

$$
\hat{G}_{A B}|\psi\rangle=\lambda^{3 / 2}(|10\rangle-|01\rangle)+O\left(\lambda^{2}\right) .
$$

We can see that in the limit $\lambda \ll 1$ we again extract one ebit of entanglement, although now in a slightly different form of a single photon split among the two modes A and B. The success probability of the scheme scales as
$P_{\text {succ }} \propto \lambda^{3}$ for small $\lambda$. In comparison with the presqueezing strategy the success rate is thus smaller by a factor of $\lambda$. Nonetheless, the simplicity of displacement operation compensates for this reduced efficiency.

Note that the local photon subtractions combined with displacements can be seen as a local version of the nonlocal coherent photon subtraction demonstrated by Ourjoumtsev et al. [8]. There the two beams reflected off the beam splitters $\mathrm{BS}_{A}$ and $\mathrm{BS}_{B}$ were combined on another balanced beam splitter and a click of an APD placed on one of its output ports heralded coherent photon subtraction associated with the filter $\hat{G}_{A B, \mathrm{nl}}=\hat{a}+\hat{b}$. Surprisingly, the present local scheme becomes equivalent to that non-local strategy in the limit of small $\lambda$ and for carefully tuned local displacements (10). Note also that the displacements can be equivalently performed on the ancilla input ports, e.g. by injecting weak coherent states there, see Fig. 2(c). Yet another possibility is to displace the reflected beam right before the single photon detection, c.f. Fig. 2(d). This latter scheme has been recently successfully experimentally utilized to generate qubit states formed by squeezed superpositions of vacuum and single-photon states $[16$.

Let us now analyze the performance of the displacement-enhanced entanglement concentration scheme beyond the limit of $\lambda \ll 1$. In our model we take into account a non-zero reflectance $R$ of the beam-splitters $\mathrm{BS}_{A}$ and $\mathrm{BS}_{B}$ and we assume on-off single-photon detectors described by a two-component POVM $\hat{\Pi}_{0}=|0\rangle\langle 0|$ and $\hat{\Pi}_{1}=\hat{I}-\hat{\Pi}_{0}$, where $\hat{I}$ denotes the identity operator and $\hat{\Pi}_{1}$ corresponds to a click of the the detector. For the sake of simplicity we assume that the detectors have unit efficiency. Experiments usually involve highly unbalanced beam splitters with $R \ll 1$. In this limit, the main effect of non-unit detection efficiency $\eta$ is the reduction of success rate by a factor of $\eta^{2}$. More specifically, the entanglement concentration scheme with inefficient detectors is equivalent to a scheme with perfect detectors and reduced reflectance of the beam splitters, $R^{\prime}=\eta R /[1-(1-\eta) R]$, preceded by the transmission of the state through a lossy channel with transmittance $\tilde{T}=1-(1-\eta) R$. In the limit of $R \ll 1$ the latter can be neglected since $\tilde{T} \approx 1$. We allow for an arbitrary initial mixed two-mode Gaussian state $\hat{\rho}_{\text {in }}$ as we shall investigate entanglement concentration of two-mode squeezed vacuum states transmitted over lossy channels. We denote by $\hat{U}_{\mathrm{BS}, A C}$ and $\hat{U}_{\mathrm{BS}, B D}$ the unitary operation describing mixing of two modes on the unbalanced beam splitters with reflectance $R$. The non-normalized density matrix of the concentrated state can be expressed as follows [25]

$\hat{\rho}_{\text {out }}=\operatorname{Tr}_{C D}\left[\left(\hat{\Pi}_{C D} \otimes \hat{I}_{A B}\right) \hat{U}\left(\hat{\rho}_{\text {in }, A B} \otimes|00\rangle\left\langle\left. 00\right|_{C D}\right) \hat{U}^{\dagger}\right]\right.$,

where

$$
\hat{U}=\hat{U}_{\mathrm{BS}, A C} \otimes \hat{U}_{\mathrm{BS}, B D} \hat{D}_{A}(\alpha) \otimes \hat{D}_{B}(\beta) \otimes \hat{I}_{C D},
$$

$\hat{\Pi}_{C D}=\hat{\Pi}_{1, C} \otimes \hat{\Pi}_{1, D}$, and $\operatorname{Tr}_{C D}$ denotes a partial trace 

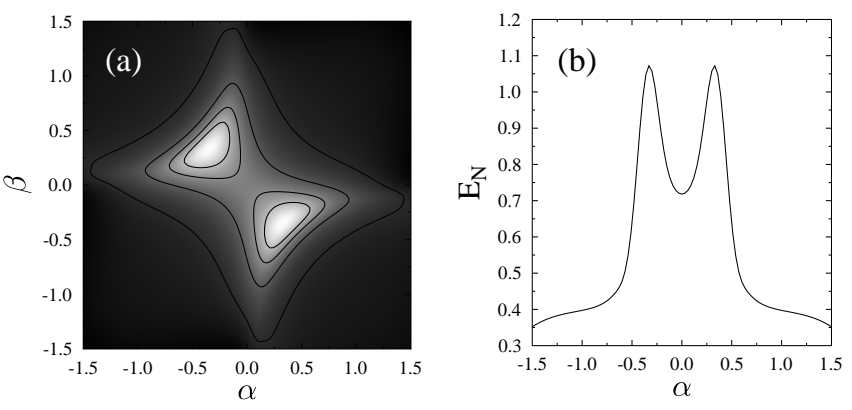

FIG. 3: Logarithmic negativity $E_{N}$ of two-mode squeezed state after entanglement concentration. Contour plot in panel (a) shows dependence of $E_{N}$ on the local displacements $\alpha$ and $\beta$ and panel (b) represents the cut through panel (a) along the diagonal line $\beta=-\alpha$. The parameters read $\lambda=0.15$ and $R=0.1$.

over modes $\mathrm{C}$ and $\mathrm{D}$ that are initially prepared in the vacuum state $|00\rangle_{C D}$.

We quantify the entanglement of the concentrated state $\hat{\rho}_{\text {out }}$ by logarithmic negativity $E_{N}$, which is a computable measure of entanglement for non-Gaussian mixed states [29]. Note that $\hat{\rho}_{\text {out }}$ is mixed even for pure input $\hat{\rho}_{\text {in }}$ due to the inability of the APDs to resolve number of photons. Recall that

$$
E_{N}\left(\hat{\rho}_{A B}\right)=\log _{2}\left\|\hat{\rho}_{A B}^{T_{A}}\right\|_{1},
$$

where $T_{A}$ denotes partial transposition with respect to mode $\mathrm{A}$ and $\|\hat{X}\|_{1}=\operatorname{Tr} \sqrt{\hat{X}^{\dagger} \hat{X}}$. In order to calculate $E_{N}$ we need to determine matrix elements of $\hat{\rho}_{\text {out }}$ in Fock state basis. One option is to explore the fact that for a Gaussian input state $\hat{\rho}_{\text {in }}$ the output state $\hat{\rho}_{\text {out }}$ can be expressed as a linear combination of four Gaussian states [15, 25, 30]. For a Gaussian state, the density matrix elements in Fock basis can be expressed in terms of multivariate Hermite polynomials because the Husimi Q-function of the state, which is a generating function of these matrix elements, has a Gaussian form. Here we follow a more straightforward route and directly evaluate Eq. (12) in Fock basis truncated at Fock state $n_{\max }=10$. For the considered values of $\lambda$ and other parameters, the errors due to the truncation are negligible.

In Fig. 3 we plot the dependence of logarithmic negativity of concentrated state on the local displacements $\alpha$ and $\beta$ for initial pure two-mode squeezed vacuum with $\lambda=0.15$ and beam splitters with $10 \%$ reflectance, $R=0.1$. We can see that the two peaks of maximum entanglement lie on the line $\alpha=-\beta$, as expected from the symmetry. Numerical calculations indicate that it suffices to consider real displacements, adding complex phase factors does not further increase the entanglement. In the rest of the paper we thus restrict ourselves to real $\alpha$ and always set $\beta=-\alpha$.

The dependence of the performance of entanglement concentration protocol on the two-mode squeezing constant $\lambda$ is illustrated in Fig. 4. The logarithmic negativities of an initial pure two-mode squeezed vacuum state, a state after local single-photon subtractions, and a state after local single-photon subtractions combined with optimal local displacements are shown in Fig. 4(a). We can see that the scheme with local displacements outperforms the scheme involving only photon subtractions and it yields almost 1 e-bit of entanglement even in the limit $\lambda \ll 1$. The slight reduction of $E_{N}$ below 1 e-bit is caused by on-off single-photon detectors leading to mixed output state after entanglement concentration. The data plotted in Fig. 4(b) confirm that the success probability of the protocol scales as $R^{2} \lambda^{3}$ (with displacements) and $R^{2} \lambda^{2}$ (without displacements). The optimal displacement $\alpha_{\mathrm{opt}}$ follows the scaling $\alpha_{\mathrm{opt}}=\sqrt{\lambda}$ for small $\lambda$, c.f. Fig. $4(\mathrm{c})$, but for larger $\lambda$ it holds that $\alpha_{\text {opt }}<\sqrt{\lambda}$.

Finally we investigate entanglement concentration of mixed states obtained by transmitting each mode of a pure two-mode squeezed vacuum state through a lossy channel with transmittance $1-\nu$. Results of numerical simulations plotted in Fig. 5 reveal that the local displacements help to increase the attainable entanglement even in the presence of losses although the achievable entanglement decreases with increasing channel losses $\nu$. Also the success probability of concentration and the optimal displacement decrease with increasing losses, see Fig. 5(b) and 5(c). This effect can be attributed to signal attenuation and reduction of mean number of photons due to transmission through a lossy channel.

\section{CONCLUSIONS}

In summary we have investigated continuous-variable entanglement concentration schemes based on local photon subtractions combined with local Gaussian opera-
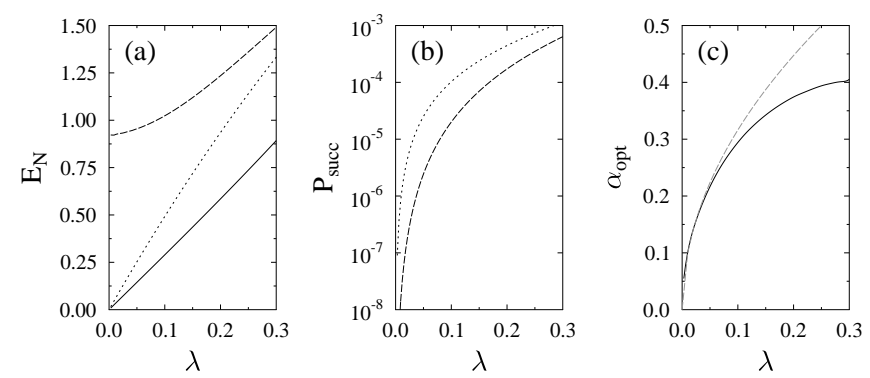

FIG. 4: Performance of entanglement concentration in dependence on the two-mode squeezing constant $\lambda$. In panel (a) we plot the logarithmic negativities $E_{N}$ of three states: initial pure two-mode squeezed vacuum (solid line), a twomode state after local photon subtractions (dotted line) and a two-mode state after local photon subtractions combined with optimal local displacements (dashed line). Panel (b) shows the success rates $P_{\text {succ }}$ of the concentration protocol with (dashed line) and without (dotted line) displacements. Panel (c) shows the optimal displacement $\alpha_{\text {opt }}$ (solid black line) and the $\sqrt{\lambda}$ reference curve for comparison (gray dashed line). The results were obtained for $R=0.1$. 

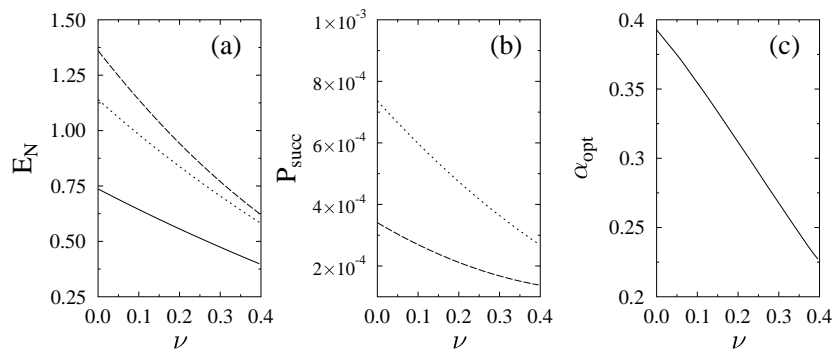

FIG. 5: Entanglement concentration of a two-mode squeezed state transmitted over a lossy channel with loss factor $\nu$. Panel (a) shows the logarithmic negativities $E_{N}$ of an initial mixed two-mode squeezed state (solid line), a two-mode state after local photon subtractions (dotted line) and a two-mode state after local photon subtractions combined with optimal local displacements (dashed line). Panel (b) shows the success rates $P_{\text {succ }}$ of the concentration protocol with (dashed line) and without (dotted line) displacements. Panel (c) shows the optimal displacement $\alpha_{\mathrm{opt}}$. The other parameters read $\lambda=0.25$ and $R=0.1$.

tions. A simple analysis in Fock basis provided an insight into the working principle of the squeezing-enhanced entanglement concentration scheme proposed by Zhang and van Loock [25]. We have next shown that a similar entanglement enhancement can be achieved by employing local displacements instead of single-mode squeezing. Remarkably, the entanglement is enhanced by a destructive quantum interference that suppresses or even completely eliminates the otherwise dominant vacuum term. The displacement-based scheme is much easier to implement experimentally than the scheme with local squeezers albeit at the cost of a somewhat reduced success rate of the protocol. Note also that while the entanglement concentration schemes involving local displacement or local squeezing can produce similar amount of entanglement, the internal structure of the entangled states is rather different. In the weak-squeezing limit and up to local Gaussian operations the displacement enhanced scheme essentially yields an entangled single-photon state $\frac{1}{\sqrt{2}}(|10\rangle-|01\rangle)$ while the scheme with local squeezers produces a state exhibiting almost perfect photon number correlations $\frac{1}{\sqrt{2}}(|00\rangle+|11\rangle)$.

The analytical and numerical results indicate that the entanglement enhancement achieved by single photon subtractions combined with optimal local displacements is of the order of $1 \mathrm{e}$-bit. Here the entanglement enhancement is defined as a difference between entanglement of concentrated and input states. Even higher entanglement enhancement could be achieved by more sophisticated local filtering operations such as the recently proposed probabilistic noiseless amplifier that approximates the unphysical operation $g^{\hat{n}}$, where $g>1$ is the amplification gain [18 24]. Such amplifier may in principle generate arbitrarily large amount of entanglement from any initial pure two-mode squeezed vacuum state. However, the resulting scheme may become rather complex and its success rate very small.

From a more general perspective, the entanglement concentration protocol analyzed in this paper is an example of a CV quantum information processing scheme whose performance can be significantly enhanced by a suitable preprocessing with Gaussian operations. Another example of such improvement is the recently proposed interface between two weakly coupled quantum systems [31, 32] where a perfect transmission of quantum state from one system to the other can be achieved with the help of ancilla modes and auxiliary Gaussian operations. It will be interesting to investigate whether similar Gaussian pre- or post-processing may be beneficial also in other CV QIP protocols.

Note added: After this work was completed we became aware of a related paper by Lee et al. 33] where entanglement concentration of two-mode squeezed vacuum by linear combination of photon addition and subtraction is investigated. This protocol is equivalent to the protocol discussed in Sec. II of the present paper, and it is shown in Ref. [33] that one ebit of entanglement can be obtained by this method in the limit of weak squeezing.

\section{Acknowledgments}

We acknowledge the financial support of the Future and Emerging Technologies (FET) programme within the Seventh Framework Programme for Research of the European Commission, under the FET-Open grant agreement COMPAS, number 212008, co-financed by the Czech Ministry of Education (7E08028). This work was also supported by the Czech Ministry of Education under the projects Center of Modern Optics (LC06007) and Measurement and Information in Optics (MSM6198959213).
[1] J. Eisert, S. Scheel, and M.B. Plenio, Phys. Rev. Lett. 89, 137903 (2002).

[2] G. Giedke and J.I. Cirac, Phys. Rev. A 66, 032316 (2002).

[3] J. Fiurášek, Phys. Rev. Lett. 89, 137904 (2002).

[4] L.M. Duan, G. Giedke, J.I. Cirac, and P. Zoller, Phys. Rev. Lett. 84, 4002 (2000).

[5] T. Opatrný, G. Kurizki, and D.-G. Welsch, Phys. Rev.
A 61, 032302 (2000).

[6] P. T. Cochrane, T. C. Ralph, and G. J. Milburn, Phys. Rev. A 65, 062306 (2002).

[7] S. Olivares, M. G. A. Paris, and R. Bonifacio, Phys. Rev. A 67,032314 (2003).

[8] A. Ourjoumtsev, A. Dantan, R. Tualle-Brouri, and P. Grangier, Phys. Rev. Lett. 98, 030502 (2007).

[9] H. Takahashi, J.S. Neergaard-Nielsen, M. Takeuchi, M. 
Takeoka, K. Hayasaka, A. Furusawa, and M. Sasaki, Nature Photonics 4, 178 (2010).

[10] S.L. Zhang and P. van Loock, Phys. Rev. A 82, 062316 (2010).

[11] A. Ourjoumtsev, R. Tualle-Brouri, J. Laurat, and Ph. Grangier, Science 312, 83 (2006).

[12] J.S. Neergaard-Nielsen, B.M. Nielsen, C. Hettich, K. Molmer, and E.S. Polzik, Phys. Rev. Lett. 97, 083604 (2006).

[13] K. Wakui, H. Takahashi, A. Furusawa, and M. Sasaki, Opt. Express 15, 3568 (2007).

[14] T. Gerrits, S. Glancy, T.S. Clement, B. Calkins, A.E. Lita, A.J. Miller, A.L. Migdall, S.W. Nam, R.P. Mirin, and E. Knill, Phys. Rev. A 82, 031802(R) (2010).

[15] J. Fiurášek, R. García-Patrón, and N.J. Cerf, Phys. Rev. A 72, 033822 (2005).

[16] J.S. Neergaard-Nielsen, M. Takeuchi, K. Wakui, H. Takahashi, K. Hayasaka, M. Takeoka, and M. Sasaki, Phys. Rev. Lett. 105, 053602 (2010).

[17] P. Marek and J. Fiurášek, Phys. Rev. A 82, 014304 (2010).

[18] T.C. Ralph and A.P. Lund, in: Proc. of the Ninth International Conference on Quantum Communication, Measurement, and Computing, Calgary, Canada, August 924, 2008 (QCMC 2008), p. 155.

[19] P. Marek and R. Filip, Phys. Rev. A 81, 022302 (2010).

[20] J. Fiurášek, Phys. Rev. A 80, 053822 (2009).
[21] G.Y. Xiang, T.C. Ralph, A.P. Lund, N. Walk, and G.J. Pryde, Nature Phot. 4, 316-319 (2010).

[22] F. Ferreyrol, M. Barbieri, R. Blandino, S. Fossier, R. Tualle-Brouri, and P. Grangier, Phys. Rev. Lett. 104, 123603 (2010).

[23] M.A. Usuga, C.R. Muller, C. Wittmann, P. Marek, R. Filip, C. Marquardt, G. Leuchs, and U.L. Andersen, Nature Phys. 6, 767 (2010).

[24] A. Zavatta, J. Fiurášek, and M. Bellini, Nature Phot. 5, 52 (2011).

[25] S.L. Zhang and P. van Loock, arXiv:1103.4500 (2011).

[26] R. Filip, P. Marek, and U.L. Andersen, Phys. Rev. A 71, 042308 (2005).

[27] J.-I. Yoshikawa, T. Hayashi, T. Akiyama, N. Takei, A. Huck, U.L. Andersen, and A. Furusawa, Phys. Rev. A 76, 060301(R) (2007).

[28] C.H. Bennett, H.J. Bernstein, S. Popescu, and B. Schumacher, Phys. Rev. A 53, 2046 (1996).

[29] G. Vidal and R.F. Werner, Phys. Rev. A 65, 032314 (2002).

[30] R. García-Patrón, J. Fiurášek, and N.J. Cerf, Phys. Rev. A 71, 022105 (2005).

[31] R. Filip, Phys. Rev. A 80, 022304 (2009).

[32] P. Marek and R. Filip, Phys. Rev. A 81, 042325 (2010).

[33] S.-Y. Lee, S.-W. Ji, H.-J. Kim, and H. Nha, Phys. Rev. A 84, 012302 (2011). 